\title{
A Study on TCP/IP Network-based Embedded Linux Intelligent System
}

\author{
Lili Fan \\ Experimental cente, Jiujiang University, Jiujiang, 332005, China
}

Key words: Embedded, Linux operating system, Monitor.

\begin{abstract}
With the development of embedded technology, we are supposed to further develop embedded monitoring system of low cost, which attracts much attention. This system, however, is not suitable for household application from the aspect of cost. In view of this, we designed an approach of realizing monitoring via capturing pictures and video stream with USB camera based on ARM9 platform. This approach has advantages of low cost and high performance, so it can be widely used for anti-theft alarm, video door bell, etc.
\end{abstract}

\section{Introduction}

We tended to think that a monitoring system is sold at a high price and has a high configuration. With the continuous advancement of IT technology, monitoring system constantly develops, and some intelligent systems sold more than RMB1000 draw extensive attention and are widely used in video door bell, etc. In this paper, based on market development needs, a household control system of low cost was designed. The working principle of this system is: pictures are obtained via an ordinary USB camera, stored in a USB flash disk, and viewed through a PC. The pictures are so clear that the resolution ratio can reach $640 * 480$ at the most. The videos run smoothly, of which the brightness and contrast ratio are adjustable. Comparing with ordinary equipment, this system costs lower and is easier to operate. This is because its size is small[5]. With a video monitor, you can obtain the pictorial information of the targets, monitor and record video image, take action according to the pictorial information, and realize monitoring and keeping watch for the targets. Video monitor is widely used in military field, customs and public security. With technical advancement and cost reduction, it is also widely used in household. In this paper, we designed an approach of realizing monitoring via capturing pictures and video stream with USB camera based on ARM9 platform, which is of great importance and effect.

\section{TCP/IP Protocol Analysis}

TCP/IP protocol is the basis of the internet, and also the basic knowledge required for protocol analysis-based intrusion detection system. The following is a brief introduction. It is a network research project launched in the late 1960s funded by American government, and developed into a common network type in the 1990s. It has an open system, and is available without time consumption, so it is called world wide web. The WAN (wide area network) has become the largest computer network in the world. TCP/IP protocol has four hierarchies, as shown in Tab. 1: 
Tab. 1 TCP/IP Protocol Family and Hierarchical Structure

\begin{tabular}{|c|c|c|c|c|c|}
\hline $\begin{array}{c}\text { Application } \\
\text { Layer }\end{array}$ & $\begin{array}{c}\text { SMTP (Simple } \\
\text { Message } \\
\text { Transfer } \\
\text { Protocol) }\end{array}$ & $\begin{array}{l}\text { HTTP (Hyper } \\
\text { Text Transport } \\
\text { Protocol) }\end{array}$ & $\begin{array}{c}\text { FTP (File } \\
\text { Transfer } \\
\text { Protocol) }\end{array}$ & $\begin{array}{l}\text { DNS (Domain } \\
\text { Name System) }\end{array}$ & $\begin{array}{c}\text { SNMP } \\
\text { (Simple } \\
\text { Network } \\
\text { Management } \\
\text { Protocol) } \\
\end{array}$ \\
\hline $\begin{array}{c}\text { Transport } \\
\text { Layer }\end{array}$ & \multicolumn{3}{|c|}{ TCP (Transmission Control Protocol) } & \multicolumn{2}{|c|}{ UDP (User Datagram Protocol) } \\
\hline $\begin{array}{c}\text { Network } \\
\text { Layer }\end{array}$ & \multicolumn{5}{|c|}{ IP, ICMP, ARP, RARP } \\
\hline $\begin{array}{c}\text { Network } \\
\text { Interface } \\
\text { Layer } \\
\end{array}$ & Ethernet & \multicolumn{2}{|c|}{ Token-Ring } & 100BASE-T & Others \\
\hline
\end{tabular}

\section{System Hardware Design}

\section{General Arrangement of System Hardware}

The system hardware has realized the purpose of typewriting in HHARM2410. This platform is provided with a USB interface, but our system needs two interfaces. One is for image collection, which can be used for USB flash disk. In view of this, images can be stored in a USB flash disk first. Meanwhile, a USB interface is also needed. Besides, we need a small infrared panel to control collection. The infrared heating system is provided with an infrared sensor and a relay. For purpose of USB camera, Zc301P chip with the highest resolution ratio was employed. S3C2410 has been set with a bus controller, and will not have external expansion. System modulation is realizable through the window to smoothen data transmission of network video. Alternatively, file download or adjustment can be made.

The allocation of peripheral resources of the system is as shown in Tab. 2.

Tab. 2 Peripheral Resource Allocation of System

\begin{tabular}{|c|c|c|c|}
\hline Peripheral Device & Function & Bank Space & Remarks \\
\hline K4S561632C & $\begin{array}{c}\text { SDRAM, system } \\
\text { internal storage }\end{array}$ & Bank0 & Capacity 64MB \\
\hline TE28F640 & $\begin{array}{c}\text { FLASH, internal } \\
\text { memory }\end{array}$ & Bank6 & Bank1 \\
\hline DM9000 & Network chip & & $\begin{array}{c}\text { External interruption } \\
\text { EINT0 }\end{array}$ \\
\hline MAX3232 & Serial chip & $\begin{array}{c}\text { Process infrared signal } \\
\text { jointly with BISS0001, } \\
\text { use GPI0 GPF2 }\end{array}$ \\
\hline PD632 & Electric infrared sensor & & \\
\hline BISS0001 & Sensor signal processor & & \\
\hline
\end{tabular}

\section{Embedded System Software Development Process}

\section{Set up the development environment of host machine}

Many linux software can be realized in a native mode, namely native development, debugging and run. Native mode is not extensively used in embedded systems, because embedded systems don't have enough resources, and are capable of debugging and running development tool. If an ordinary embedded system adopts cross compiling, then the native mode can be established in the host. Developer edition is also called target edition. 
For purpose of development, cross compiling may be adopted, together with tool, to generate a type of binary code, which can be downloaded from file or run in the target host. For different rentals, target formats, and hosts with Intel processor or AMD processor, GNU (a compiler) has different functions. Thus, a specific host and target machine should be selected for compiling, so as to create a better development environment, and a host with an operating system (Linux) is needed for embedded development. There are multiple versions of Linux. We chose Red Hat Linux 9.0 here.

If a computer is required to be installed with windows and Linux, this computer will be started up frequently. But this may impact the work environment. Thus, the installation of windows can be realized via installing virtual software. The installation of virtual Linux system is same with that of real Linux system. It's unnecessary to go into details. Besides, we can install all kinds of software to facilitate our using system service in future. We also should learn to set rules for configuring the firewall.

After installation is finished, a large hard-disk space of the system may be occupied. Therefore, we should think about entering $\mathrm{X}$ mode or text system after starting up the Linux system. After the completion, further modification is still needed, so as to determine to enter windows or text system. This is of great help. In windows system, the emergence of inexecutable unmount file system will be avoided, because the fam monitor program may collide with windows system. VMware excludes any serial port. Therefore, serial port should be installed, otherwise the information of the developer edition cannot be displayed. After that, it is required to create an environment for debugging various films. After the completion of environmental development, subdirectory can be generated under the root directory.

\section{Samba Server Configuration}

Samba realized file sharing with Linux and Windows, can realize print sharing, and can install a server under Linux system, so that users can get a safe access to the file system.

You may install various compilation tools under Linux system, but none of them is better than the Source Insight of windows. Source Insight is capable of program analysis, analyzing source code during running, maintaining its own symbol database, drawing useful contextual information, and distinguishing variables and function name based on different colors. In a word, Source Insight is a successfully-developed large software. So we should replace the editing tools under Linux with Source Insight. But Source Insight can run in the environment of windows system. The function of file sharing of Samba will help us to overcome this defect. We can modify programs under Linux via editing Source Insight, and perform direct compilation with cross compilation tools, favorable to system debugging and shortening software development period.

\section{Application Software Development}

If the host is connected to the target edition via the Ethernet port, then minicom can be run on the host as the display terminal of the target edition. Besides, the mount host hard disk can be acquired on the target edition, to realize debugging application program on the target edition.

\section{System Debugging and Computational Results}

\section{Start-up Procedure of ARM Linux}

To start up ARM Linux, a program should be run in the kernel, which is helpful to initialize equipment and call Linux to transmit kernel information. Generally speaking, the smallest bootloader can obtain the following functions:
a. Realize and perform RAM initialization
b. Initialize a serial port
c. Check machine type
d. Determine kernel start-up parameter list
e. Use kernel in the form of mapping 


\section{Image and Video Collection Program Debugging}

After the arm panel starts Linux panel, install camera, GPIO and key drive (spca5xx.o, gpio_test.o and key4.o have been incorporated into the root file system/modules directory), create gpiotest and keys4 node, install USB flash disk, and run the following commands:

\#insmod/modules/spca5xx.o

\#insmod/modules/gpio_test.o

\#insmod/modules/key4.o

\#mknod/dev/gpiotest c 2210

\#mknod/dev/keys4 c 1380

\#mount-t vfat/dev/sda1/tmp

These commands can be written in file rc.local script, to directly execute system power system. The collected image is as shown in Fig. 2.

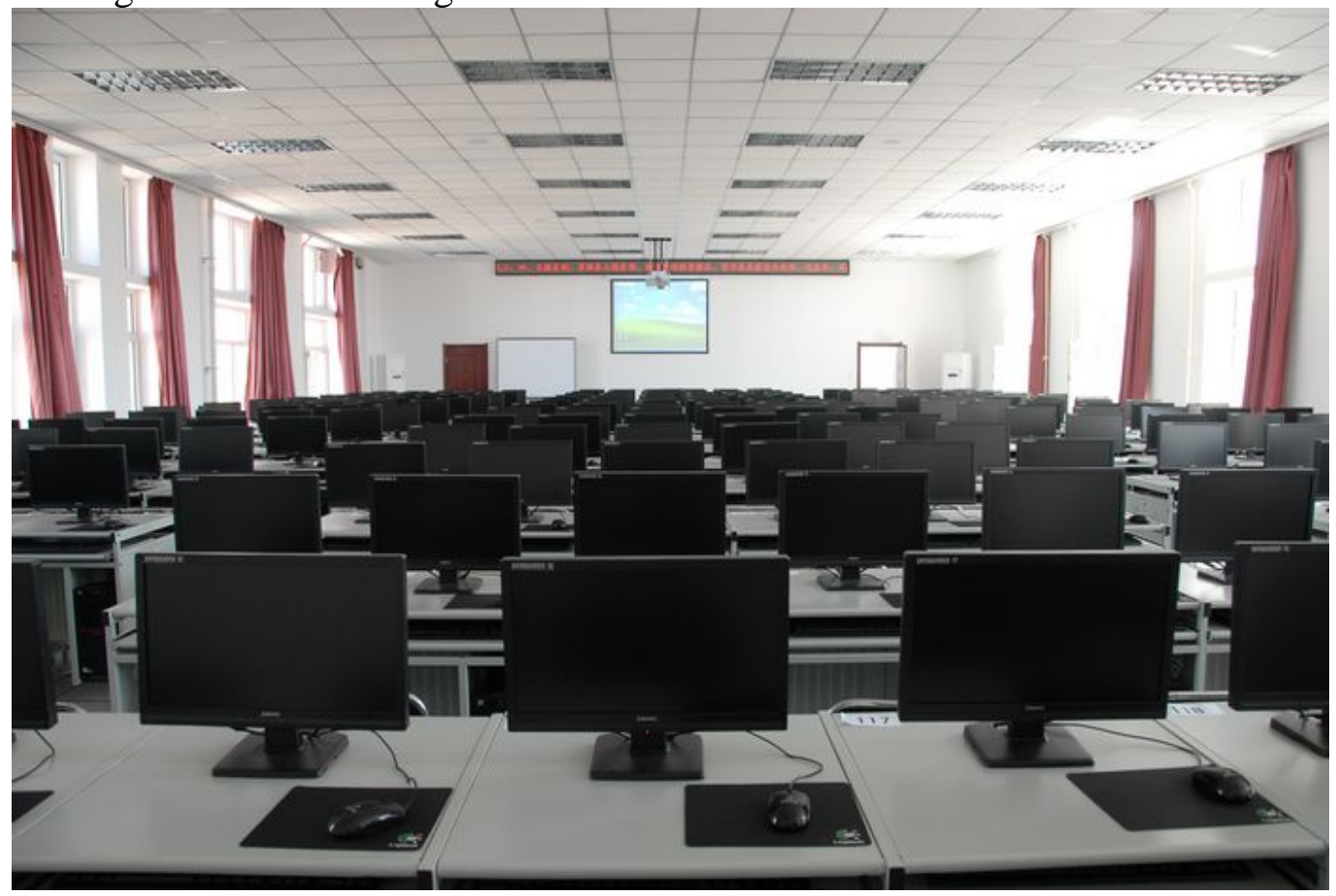

Fig. 2 Collected Image

\section{Network Monitoring Program Debugging}

Network monitoring program can run on arm panel, copy the files of "control.jpg", "JWebcamPlayer.jar" and "index.html" under the directory of http-java-applet of client's file to the file system of arm panel, and add some permission to the files. We can join arm panel and LAN, so that the host of LAN can access to arm panel via boa web server.

\section{Conclusion}

In this paper, Video4Linux API function under Linux was employed to collect video image program; image collection was realized by means of mmap, which helps to raise the efficiency of collection, and facilitates timing collection and pyroelectric infrared collection. Timing collection and pyroelectric infrared collection can be called artificial collection, and can let human enter the region for purpose of collection so as to realize intelligent control.

\section{References}


[1] Xu Lunfeng, Xiong Guangze. Real-time Operating System and Its Development Process, Journal of Computer Applications, 2015(06):56-61

[2] Liu Zhichen. Real-time Analysis and Improvement Research of An Embedded Operating System. Hunan: Hunan University 2014

[3] Zhao Yiwei, Zhang Lifen, Chen Shuoying. Design and Realization of A Real-time Operating System. Transactions of Beijing Institute of Technology, 2011. (21):196-100.

[4] Yao Paul, Windows CE3.0:Enabled Real-Time Features Provide Sophisticated Thread Handling, MSDN Magazine November. 2010.Volume 1.

[5] Gao Feng. A Core Research on Embedded Real-time Multitask Microkernel. Chengdu: University of Electronic Science and Technology of China, 2014 